\title{
Evanescent Field Enhancement in Liquid Crystal Optical Fibers: A Field Characteristics Based Analysis
}

\author{
P. K. Choudhury \\ Institute of Microengineering and Nanoelectronics (IMEN), Universiti Kebangsaan Malaysia (UKM), 43600 Bangi, Selangor, Malaysia \\ Correspondence should be addressed to P. K. Choudhury; pankaj@ukm.my
}

Received 11 July 2012; Accepted 6 February 2013

Academic Editor: Durga Ojha

Copyright ( 2013 P. K. Choudhury. This is an open access article distributed under the Creative Commons Attribution License, which permits unrestricted use, distribution, and reproduction in any medium, provided the original work is properly cited.

\begin{abstract}
The paper presents the analysis of the electromagnetic wave propagation through liquid crystal optical fibers (LCOFs) of two different types-conventional guides loaded with liquid crystals (addressed as LCOFs) and those with additional twists due to conducting helical windings (addressed as HCLCOFs). More precisely, the three-layer optical waveguide structures are considered along with its outermost region being loaded with radially anisotropic liquid crystal material and the inner regions being made of usual silica, as used in conventional optical fibers. In addition to that, LCOF with twists introduced in the form of conducting helical windings at the interface of the silica core and the liquid crystal clad is also taken into account. Emphasis has been put on the power confinements by the lower-order TE modes sustained in the different sections of the LCOF structure. The results demonstrate useful applications of these guides in integrated optics as the power sustained in the liquid crystal section by the excited TE modes remains very high. In the case of twisted clad liquid crystal guides, descriptions are limited to the nature of dispersion relation only under the TE mode excitation, and corresponding to the cases of helix orientations being parallel and perpendicular to the optical axis.
\end{abstract}

\section{Introduction}

Optical fibers are of immense use in communication technology, which may be either for the long distance use, for example telecommunication needs, or the short distance ones, for example sensing and so forth. In either case, optical fibers can be designed to act as active and/or passive device. The propagation characteristics of various types of optical fibers have been discussed in the literature. In this respect, the fiber itself may either have wide varieties of geometries ranging from conventional to the unconventional ones, or the guide may possess different forms of material compositions. The characteristics of electromagnetic wave propagation through optical fibers are greatly dependent on the type of material which the guide is composed of.

Among the others, fibers made of liquid crystals [13] fall into the category of complex mediums and proved to be greatly attractive for multifarious applications $[4,5]$ because these materials present the distinct characteristic of anisotropic nature in their physical properties $[6,7]$. Investigators have reported liquid crystal optical fibers (LCOFs) of different forms of constructions in respect of geometry as well as material distributions. LCOFs may consist of liquid crystals either in the core [8-10] or in the clad section [911] of the guide. High optical anisotropy of liquid crystals makes them as demanding smart materials for their usage in a wide range of applications in the area of integrated optics $[7,12]$. These can be used in electric field sensing as the macroscopic optical properties of liquid crystals can be altered under the influence of external electrical field [13]. Yoshino et al. [14] demonstrated the use of LCOFs in electric field sensing based on the inherent property of liquid crystals that these exhibit the largest electrooptic effect among known materials. In all the aforesaid applications, the clad region of the guide consists of liquid crystal. For the fabrication of directional couplers, LCOFs are used with the liquid crystal section sandwiched between two side polished fibers, and coaxial fibers are embedded in liquid crystals.

Applications of liquid crystals make it clear that these exhibit distinct feature of polarization anisotropy which may have two different directions of orientation-radial and azimuthal anisotropies; the latter one is less discussed in 
the literature. In the present paper, we consider the nematic form of liquid crystals along with its radially anisotropic nature to constitute the fiber clad, the core region being made of standard silica. In forming such LCOFs, the radial anisotropy of liquid crystal can be obtained by capillary action after inserting the liquid crystal section (of the fiber) into a capillary tube coated with N,N-dimethyl-N-octadecyl3-aminopropyltrimethoxysilyl chloride [11].

Considering the importance of the usage of liquid crystals in optical fibers, such guides with tapered structures have been discussed before [15]. Since the propagation of power through a guide plays the determining role for specific applications of the guide, the scope of the present paper lies in the analysis of LCOFs in respect of power confinement factor. We consider a three-layer LCOF, of which the outermost clad is made of radially anisotropic liquid crystal material, and determine the power propagation characteristics corresponding to two lower-order TE modes in the guides of different dimensions. It has been observed that the TE modes transport relatively high amount of power through the liquid crystal clad. These interesting features of radially anisotropic LCOFs indicate their usefulness in the fabrication of different coupling devices used in the area of integrated optics, and also, in optical sensing where the method of evanescent field absorption is primarily implemented.

The investigations are extended to the case of LCOFs with conducting helical windings (i.e., HCLCOFs-the helical clad liquid crystal optical fibers) introduced at the coreclad interface of the guide. In such fibers, the angle of pitch (of the helix in use) exhibits dominant characteristics to alter the wave propagation, and the working principles are based on the theory of low- and medium-power travelling wave tubes (TWTs) [16]. Implementing the concept in the case of dielectric optical fibers, studies have appeared in the literature for fibers having circular and elliptical crosssections [17-20]. However, in the present paper, as stated before, the existence of the liquid crystal layer will modify the propagation characteristics. The dispersion relation for such types of guides (i.e. HCLCOFs) is deduced, and the features are reported corresponding to the cases of helical turns being parallel as well as perpendicular to the optical axis.

\section{The Analytical Approach}

2.1. The Case of LCOFs. Figure 1 illustrates the cross-sectional view of the three-layer LCOF, of which the (infinitely extended) outermost clad is made of radially anisotropic liquid crystal material; the core and the inner clad regions being constructed of linear, homogeneous, and isotropic dielectrics having the refractive index (RI) values as $n_{1}$ and $n_{2}$ $\left(n_{1}>n_{2}\right)$. Also, $n_{o}$ and $n_{e}$, respectively, are the ordinary and the extraordinary RIs of the liquid crystal section. Further, $\rho_{1}$ and $\rho_{2}$ are, respectively, the radii of the core and the inner clad regions. The dashed lines in the radial direction (in Figure 1) indicate the radial orientation of liquid crystal molecules. We consider the time harmonic waves with angular frequency $\omega$ (in the unbounded medium) propagate along the $z$-direction with the propagation constant $\beta$, and the wave is harmonic

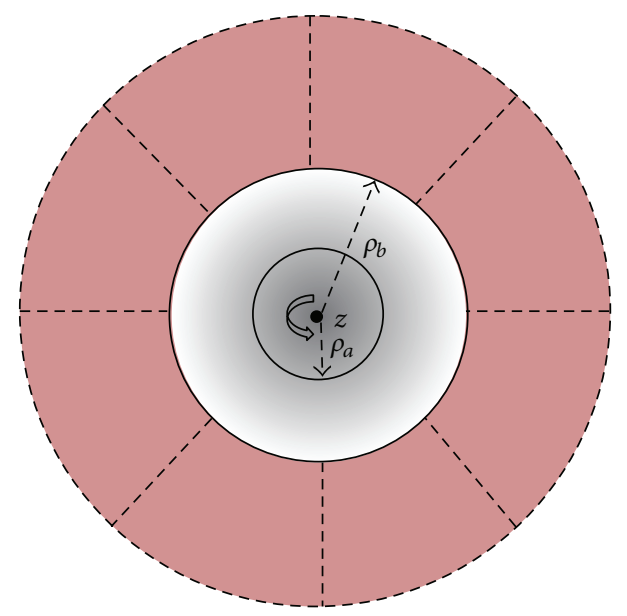

Figure 1: Cross-sectional view of a three-layer LCOF with the outermost section filled with liquid crystal (the dashed lines in the radial direction indicate the orientation of the liquid crystal molecules).

in the direction of propagation too. Of the liquid crystal section, the principal axes coincide with the $z$-axis, and the extraordinary principal axis is radially oriented. Therefore, the liquid crystal region will have the RI distribution as

$$
n_{\rho}=n_{e}, \quad n_{\phi}=n_{z} \quad \text { with } n_{e}>n_{1}>n_{2}>n_{o},
$$

where $n_{\rho}, n_{\phi}$, and $n_{z}$ are, respectively, the RI values along the $\rho$-, $\phi$-, and $z$-directions. Now, following the formulations presented in [21], the coupled wave equations for the transverse field components can be written as [22]

$$
\begin{aligned}
\left(\nabla_{t}^{2}+\right. & \left.k_{0}^{2} n_{\rho}^{2}-\beta^{2}-\frac{1}{\rho^{2}}\right) e_{\rho} \\
= & -\frac{2}{\rho^{2}} \frac{\partial e_{\phi}}{\partial \phi}+\left(1-\frac{n_{\rho}^{2}}{n_{z}^{2}}\right) \frac{\partial}{\partial \rho}\left\{\frac{1}{\rho} \frac{\partial}{\partial \rho}\left(\rho e_{\rho}\right)\right\} \\
& +\left(1-\frac{n_{\phi}^{2}}{n_{z}^{2}}\right) \frac{\partial}{\partial \rho}\left(\frac{1}{\rho} \frac{\partial e_{\phi}}{\partial \phi}\right) \\
\left(\nabla_{t}^{2}+\right. & \left.k_{0}^{2} n_{\phi}^{2}-\beta^{2}-\frac{1}{\rho^{2}}\right) e_{\phi} \\
= & \frac{2}{\rho^{2}} \frac{\partial e_{\rho}}{\partial \phi}+\frac{1}{\rho^{2}}\left\{\left(1-\frac{n_{\rho}^{2}}{n_{z}^{2}}\right) \frac{\partial}{\partial \rho}\left(\rho \frac{\partial e_{\rho}}{\partial \phi}\right)\right. \\
& \left.+\left(1-\frac{n_{\phi}^{2}}{n_{z}^{2}}\right) \frac{\partial^{2} e_{\phi}}{\partial \phi^{2}}\right\},
\end{aligned}
$$

where $\nabla_{t}^{2}$ is the Laplacian operator in the cylindrical coordinate system and $k_{0}$ is the free-space propagation constant.

Liquid crystal is anisotropic in nature, and therefore, the sustained modes in the LCOF under consideration will essentially contain the electric field components $E_{\rho}, E_{\phi}$, and $E_{z}$, each facing different RI values in their respective 
directions. However, in the present work, we consider the lower-order $\mathrm{TE}$ modes (namely, $\mathrm{TE}_{01}$ ). In this case, there is only one transverse electrical field component $e_{\phi}$, which is independent of the coordinate $\phi$, making thereby one to have $e_{\rho}=0$ and $\partial e_{\phi} / \partial \phi=0$ corresponding to this mode. Implementing these conditions and using (2a) and (2b), after some mathematical steps, it can be shown that the electric/magnetic field components in this case will be ultimately given as

$$
\begin{gathered}
\left.H_{\rho}\right)_{\mathrm{I}}=-C_{1} \frac{\beta}{\omega \mu_{0}} J_{1}\left(\gamma_{1} \rho\right) \exp \{j(\omega t-\beta z)\} \\
\left.H_{z}\right)_{\mathrm{I}}=C_{1} \frac{j}{\omega \mu_{0}}\left\{\gamma_{1} J_{1}^{\prime}\left(\gamma_{1} \rho\right)+\frac{1}{\rho} J_{1}\left(\gamma_{1} \rho\right)\right\} \exp \{j(\omega t-\beta z)\} \\
\left.H_{\rho}\right)_{\mathrm{II}}=-\frac{\beta}{\omega \mu_{0}}\left\{C_{2} K_{1}\left(\gamma_{2} \rho\right)+C_{3} I_{1}\left(\gamma_{2} \rho\right)\right\} \exp \{j(\omega t-\beta z)\} \\
\left.H_{z}\right)_{\mathrm{II}}=\frac{j}{\omega \mu_{0}}\left[C_{2}\left\{\gamma_{2} K_{1}^{\prime}\left(\gamma_{2} \rho\right)+\frac{1}{\rho} K_{1}\left(\gamma_{2} \rho\right)\right\}\right. \\
\left.+C_{3}\left\{w I_{1}^{\prime}\left(\gamma_{2} \rho\right)+\frac{1}{\rho} I_{1}\left(\gamma_{2} \rho\right)\right\} \exp \{j(\omega t-\beta z)\}\right] \\
\left.H_{\rho}\right)_{\mathrm{III}}=-C_{4} \frac{\beta}{\omega \mu_{0}} K_{1}\left(\gamma_{3} \rho\right) \exp \{j(\omega t-\beta z)\} \\
\left.H_{z}\right)_{\mathrm{III}}=C_{4} \frac{j}{\omega \mu_{0}}\left\{\gamma_{3} K_{1}^{\prime}\left(\gamma_{3} \rho\right)+\frac{1}{\rho} K_{1}\left(\gamma_{3} \rho\right)\right\} \\
\times \exp \{j(\omega t-\beta z)\} .
\end{gathered}
$$

In (3a), (3b), (3c), (3d), (3e), and (3f), the superscripts I, II, and III, respectively, represent the situations in the LCOF core, inner clad and the outer liquid crystal clad sections of the guide. Also, $\mu_{0}$ is the free-space permeability, $C_{1}, C_{2}, C_{3}$, and $C_{4}$ are the arbitrary constants to be determined by the boundary conditions, and $J(\cdot), K(\cdot)$, and $I(\cdot)$ represent Bessel and the modified Bessel functions. Further, the prime indicates the differentiation with respect to the argument, and the quantities $\gamma_{1}, \gamma_{2}$, and $\gamma_{3}$ are defined as

$$
\begin{aligned}
& \gamma_{1}=\sqrt{n_{1}^{2} k_{0}^{2}-\beta^{2}} \\
& \gamma_{2}=\sqrt{\beta^{2}-n_{2}^{2} k_{0}^{2}} \\
& \gamma_{3}=\sqrt{\beta^{2}-n_{o}^{2} k_{0}^{2}}
\end{aligned}
$$

Making use of the above-mentioned field components, as stated in (3a), (3b), (3c), (3d), (3e), and (3f) and implementing the electromagnetic boundary conditions at the layer interfaces of the LCOF, values of the arbitrary constants can be determined. By using those, the expressions corresponding to power [23] transmitted through the different sections of the LCOF can be evaluated as

$$
\begin{gathered}
P_{\text {core }}=C_{1}^{2} \frac{\pi}{\omega \mu_{0}}\left\{\gamma_{1} \int_{0}^{\rho_{1}} \rho J_{1}\left(\gamma_{1} \rho\right) J_{1}^{\prime}\left(\gamma_{1} \rho\right) d \rho\right. \\
\left.+\int_{0}^{\rho_{2}}\left\{J_{1}\left(\gamma_{1} \rho\right)\right\}^{2} d \rho\right\} \\
P_{\text {innerclad }}=C_{1}^{2} \frac{\pi}{\omega \mu_{0}}\left[\Psi ^ { 2 } \left\{\gamma_{2} \int_{\rho_{1}}^{\rho_{2}} \rho I_{1}\left(\gamma_{2} \rho\right) I_{1}^{\prime}\left(\gamma_{2} \rho\right) d \rho\right.\right. \\
\left.+\int_{\rho_{1}}^{\rho_{2}}\left(I_{1}\left(\gamma_{2} \rho\right)\right)^{2} d \rho\right\} \\
+\Omega^{2}\left\{\gamma_{2} \int_{\rho_{1}}^{\rho_{2}} \rho K_{1}\left(\gamma_{2} \rho\right) K_{1}^{\prime}\left(\gamma_{2} \rho\right) d \rho\right. \\
\left.+\int_{\rho_{1}}^{\rho_{2}}\left(K_{1}\left(\gamma_{2} \rho\right)\right)^{2} d \rho\right\} \\
+\Phi \Psi \\
\left\{2 \int_{\rho_{1}}^{\rho_{2}} K_{1}\left(\gamma_{2} \rho\right) I_{1}\left(\gamma_{2} \rho\right) d \rho\right. \\
+\gamma_{2} \int_{\rho_{1}}^{\rho_{2}} \rho K_{1}\left(\gamma_{2} \rho\right) I_{1}^{\prime}\left(\gamma_{2} \rho\right) d \rho \\
\left.\left.+\gamma_{2} \int_{\rho_{1}}^{\rho_{2}} \rho I_{1}\left(\gamma_{2} \rho\right) K_{1}^{\prime}\left(\gamma_{2} \rho\right) d \rho\right\}\right]
\end{gathered}
$$

$$
\begin{aligned}
P_{\text {outerclad }}= & C_{1}^{2} \frac{\pi}{\omega \mu_{0}}\left(\frac{\Phi K_{1}\left(\gamma_{2} \rho_{2}\right)+\Psi I_{1}\left(\gamma_{2} \rho_{2}\right)}{K_{1}\left(\gamma_{3} \rho_{2}\right)}\right)^{2} \\
\times & {\left[\int_{\rho_{2}}^{\infty}\left\{K_{1}\left(\gamma_{3} \rho\right)\right\}^{2} d \rho\right.} \\
& \left.+\gamma_{3} \int_{\rho_{2}}^{\infty} \rho K_{1}\left(\gamma_{3} \rho\right) K_{1}^{\prime}\left(\gamma_{3} \rho\right) d \rho\right],
\end{aligned}
$$

where

$$
\begin{gathered}
\Phi=\left(J_{1}\left(\gamma_{1} \rho_{1}\right)\left\{\gamma_{2} I_{1}^{\prime}\left(\gamma_{2} \rho_{1}\right)+\frac{1}{\rho_{1}} K_{1}\left(\gamma_{2} \rho_{1}\right)\right\}\right. \\
\left.-I_{1}\left(\gamma_{2} \rho_{1}\right)\left\{\gamma_{1} J_{1}^{\prime}\left(\gamma_{1} \rho_{1}\right)+\frac{1}{\rho_{1}} J_{1}\left(\gamma_{1} \rho_{1}\right)\right\}\right) \\
\times\left(K_{1}\left(\gamma_{2} \rho_{1}\right)\left\{\gamma_{2} I_{1}^{\prime}\left(\gamma_{2} \rho_{1}\right)+\frac{1}{\rho_{1}} K_{1}\left(\gamma_{2} \rho_{1}\right)\right\}\right. \\
\left.\quad-I_{1}\left(\gamma_{2} \rho_{1}\right)\left\{\gamma_{2} K_{1}^{\prime}\left(\gamma_{2} \rho_{1}\right)+\frac{1}{\rho_{1}} K_{1}\left(\gamma_{2} \rho_{1}\right)\right\}\right)^{-1}, \\
\quad\left(J_{1}\left(\gamma_{1} \rho_{1}\right)\left\{\gamma_{2} K_{1}^{\prime}\left(\gamma_{2} \rho_{1}\right)+\frac{1}{\rho_{1}} K_{1}\left(\gamma_{2} \rho_{1}\right)\right\}\right. \\
\left.-K_{1}\left(\gamma_{2} \rho_{1}\right)\left\{\gamma_{1} J_{1}^{\prime}\left(\gamma_{1} \rho_{1}\right)+\frac{1}{\rho_{1}} J_{1}\left(\gamma_{1} \rho_{1}\right)\right\}\right)
\end{gathered}
$$




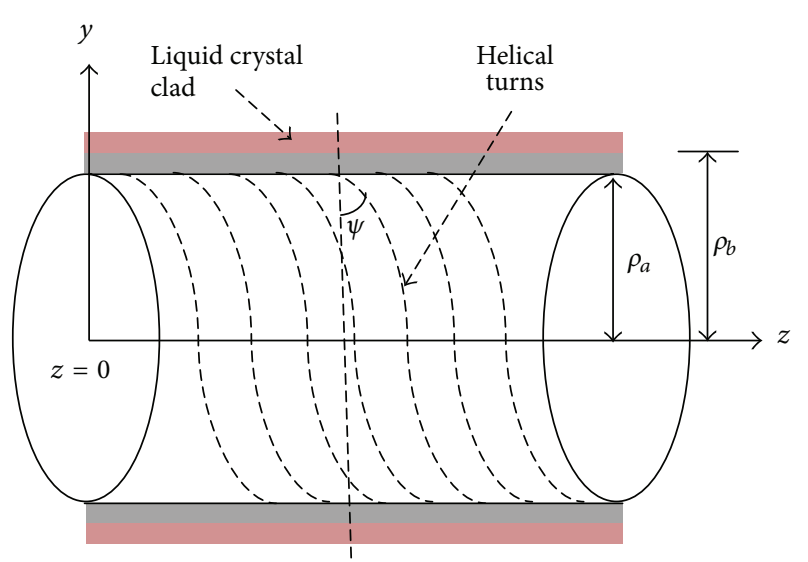

FIgURE 2: Longitudinal view of the HCLCOF under consideration.

$$
\begin{aligned}
& \times\left(I_{1}\left(\gamma_{2} \rho_{1}\right)\left\{\gamma_{2} K_{1}^{\prime}\left(\gamma_{2} \rho_{1}\right)+\frac{1}{\rho_{1}} K_{1}\left(\gamma_{2} \rho_{1}\right)\right\}\right. \\
& \left.\quad-K_{1}\left(\gamma_{2} \rho_{1}\right)\left\{\gamma_{2} I_{1}^{\prime}\left(\gamma_{2} \rho_{1}\right)+\frac{1}{\rho_{1}} K_{1}\left(\gamma_{2} \rho_{1}\right)\right\}\right)^{-1} .
\end{aligned}
$$

Above derived (5), (6), and (7), respectively, represent the expressions of the power propagating through the LCOF core, inner dielectric clad, and the outer liquid crystal clad regions. It is to be noted at this point that the evaluation of power requires the values of all the arbitrary constants $C_{1}, C_{2}, C_{3}$, and $C_{4}$ to be written in terms of only one constant (say $C_{1}$ ), which can be determined by a normalization condition taking into consideration the input power. If $P_{\text {total }}$ represents the total power transmitted through the LCOF by the $\mathrm{TE}_{01}$ modes, that is,

$$
P_{\text {total }}=P_{\text {core }}+P_{\text {innerclad }}+P_{\text {outerclad }} \text {, }
$$

then $P_{\text {core }} / P_{\text {total }}\left(\equiv \Gamma_{\text {core }}\right), P_{\text {innerclad }} / P_{\text {total }}\left(\equiv \Gamma_{\text {innerclad }}\right)$, and $P_{\text {outerclad }} / P_{\text {total }}\left(\equiv \Gamma_{\text {outerclad }}\right)$ will, respectively, determine the relative power (or the power confinement factor) in the LCOF core, inner clad, and the outer clad sections.

2.2. The Case of HCLCOFs. Figure 2 shows the longitudinal view of the HCLCOF under consideration, which incorporates some modifications in the previous guide structure illustrated in Figure 1 in the form of additional conducting helical windings loaded at the interface of the core and the inner dielectric clad sections. As the fiber core is wrapped with a conducting sheath helix at a constant angle (called as the helix pitch angle) $\psi$ around the core-inner clad boundary, the structure essentially has a circular cylindrical surface with high conductivity in a preferential direction. This way, helical clad fibers offer the additional parameter to suitably control the modal behaviour of the guide, that is, by varying the pitch angle of helix - an aspect of great technological importance [17-20].

Coming to the analytical steps, the field components in the different sections of the HCLCOFs will be represented by (3a), (3b), (3c), (3d), (3e), and (3f). However, the boundary conditions will be modified in this case [24], the implementation of which will finally provide an $8 \times 8$ determinant formed by the coefficients of the unknown constants. A nontrivial solution to the set of equations can be obtained if the determinant is equated to zero, that is,

$$
\begin{gathered}
\left(\begin{array}{cccccccc}
x_{1} & x_{2} & 0 & 0 & 0 & 0 & 0 & 0 \\
0 & 0 & x_{3} & x_{4} & x_{5} & x_{6} & 0 & 0 \\
x_{7} & -x_{8} & -x_{9} & -x_{10} & x_{11} & x_{12} & 0 & 0 \\
-x_{13} & x_{14} & x_{15} & x_{16} & -x_{17} & -x_{18} & 0 & 0 \\
0 & 0 & y_{1} & y_{2} & y_{3} & y_{4} & 0 & 0 \\
0 & 0 & 0 & 0 & 0 & 0 & y_{5} & y_{6} \\
0 & 0 & y_{7} & y_{8} & -y_{9} & -y_{10} & -y_{11} & y_{12} \\
0 & 0 & -y_{13} & -y_{14} & y_{15} & y_{16} & y_{17} & -y_{18}
\end{array}\right) \\
\times\left(\begin{array}{c}
A \\
B \\
C \\
D \\
E \\
F \\
G \\
H
\end{array}\right)=\left(\begin{array}{c}
0 \\
0 \\
0 \\
0 \\
0 \\
0 \\
0 \\
0
\end{array}\right) .
\end{gathered}
$$

In (11), the used symbols have their meanings as

$$
\begin{gathered}
x_{1}=\left(\sin \psi+\frac{v \beta}{\gamma_{1}^{2} \rho_{a}} \cos \psi\right) J_{v}\left(\gamma_{1} \rho_{a}\right), \\
x_{2}=\frac{i \omega \mu_{0}}{\gamma_{1}} \cos \psi J_{v}^{\prime}\left(\gamma_{1} \rho_{a}\right), \\
x_{3}=\left(\sin \psi+\frac{v \beta}{\gamma_{2}^{2} \rho_{a}} \cos \psi\right) K_{v}\left(\gamma_{2} \rho_{a}\right), \\
x_{4}=\left(\sin \psi+\frac{v \beta}{\gamma_{2}^{2} \rho_{a}} \cos \psi\right) I_{v}\left(\gamma_{2} \rho_{a}\right), \\
x_{5}=\frac{i \omega \mu_{0}}{\gamma_{2}} \cos \psi K_{v}^{\prime}\left(\gamma_{2} \rho_{a}\right), \\
x_{6}=\frac{i \omega \mu_{0}}{\gamma_{2}} \cos \psi I_{v}^{\prime}\left(\gamma_{2} \rho_{a}\right), \\
x_{7}=\left(\cos \psi-\frac{v \beta}{\gamma_{1}^{2} \rho_{a}} \sin \psi\right) J_{v}\left(\gamma_{1} \rho_{a}\right), \\
x_{8}=\frac{i \omega \mu_{0}}{\gamma_{1}} J_{v}^{\prime}\left(\gamma_{1} \rho_{a}\right), \\
x_{9}=\left(\cos \psi-\frac{v \beta}{\gamma_{2}^{2} \rho_{a}} \sin \psi\right) K_{v}\left(\gamma_{2} \rho_{a}\right), \\
x_{10}=\left(\cos \psi-\frac{v \beta}{\gamma_{2}^{2} \rho_{a}} \sin \psi\right) I_{v}\left(\gamma_{2} \rho_{a}\right),
\end{gathered}
$$




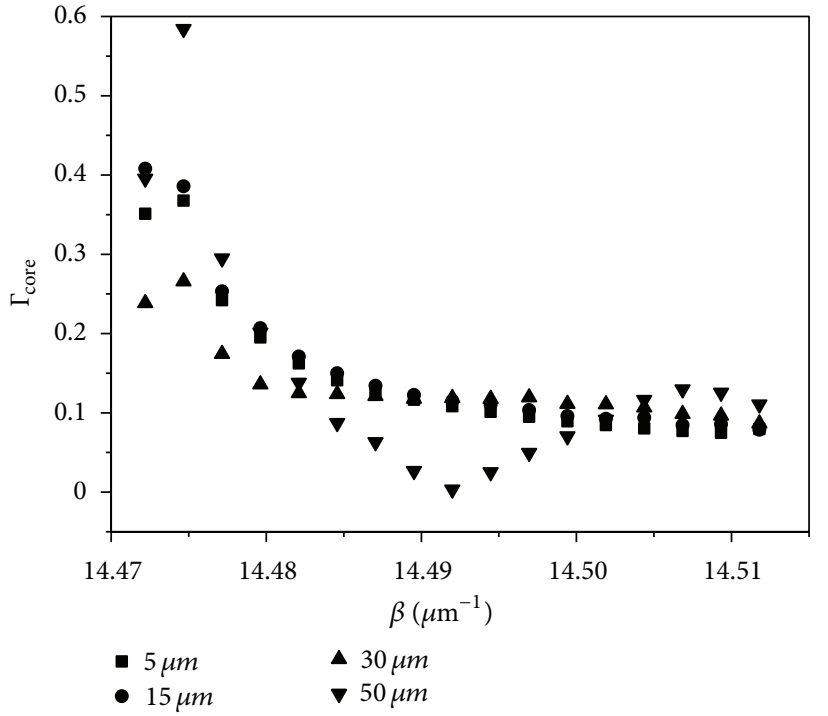

(a)

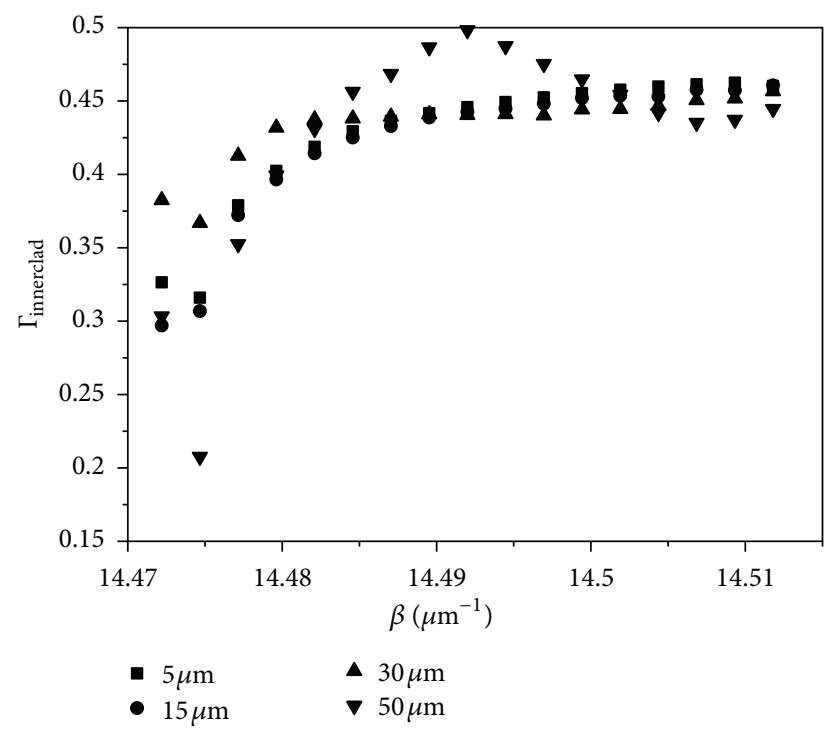

(b)

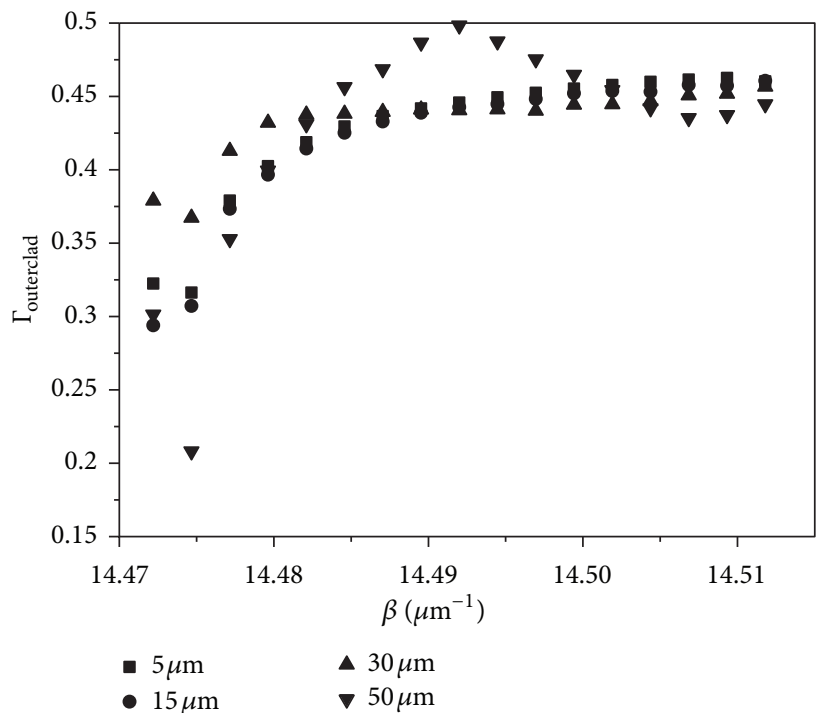

(c)

FIgure 3: (a) Power confinement by the TE modes in the LCOF core. (b) Power confinement by the TE modes in the LCOF inner clad. (c) Power confinement by the TE modes in the LCOF outer clad.

$$
\begin{array}{cc}
x_{11}=\frac{i \omega \mu_{0}}{\gamma_{2}} \sin \psi K_{v}^{\prime}\left(\gamma_{2} \rho_{a}\right), & x_{17}=\left(\sin \psi+\frac{v \beta}{\gamma_{2}^{2} \rho_{a}} \cos \psi\right) K_{v}\left(\gamma_{2} \rho_{a}\right), \\
x_{12}=\frac{i \omega \mu_{0}}{\gamma_{2}} \sin \psi I_{v}^{\prime}\left(\gamma_{2} \rho_{a}\right), & x_{18}=\left(\sin \psi+\frac{v \beta}{\gamma_{2}^{2} \rho_{a}} \cos \psi\right) I_{v}\left(\gamma_{2} \rho_{a}\right), \\
x_{13}=\frac{i \omega \varepsilon_{1}}{\gamma_{1}} \cos \psi J_{v}^{\prime}\left(\gamma_{1} \rho_{a}\right), & y_{1}=\left(\sin \psi+\frac{v \beta}{\gamma_{2}^{2} \rho_{b}} \cos \psi\right) K_{v}\left(\gamma_{2} \rho_{b}\right), \\
x_{14}=\left(\sin \psi+\frac{v \beta}{\gamma_{1}^{2} \rho_{a}} \cos \psi\right) J_{v}\left(\gamma_{1} \rho_{a}\right), & y_{2}=\left(\sin \psi+\frac{v \beta}{\gamma_{2}^{2} \rho_{b}} \cos \psi\right) I_{v}\left(\gamma_{2} \rho_{b}\right), \\
x_{15}=\frac{i \omega \varepsilon_{2}}{\gamma_{2}} \cos \psi K_{v}^{\prime}\left(\gamma_{2} \rho_{a}\right), & y_{3}=\frac{i \omega \mu_{0}}{\gamma_{2}} \cos \psi K_{v}^{\prime}\left(\gamma_{2} \rho_{b}\right), \\
x_{16}=\frac{i \omega \varepsilon_{2}}{\gamma_{2}} \cos \psi I_{v}^{\prime}\left(\gamma_{2} \rho_{a}\right), & y_{4}=\frac{i \omega \mu_{0}}{\gamma_{2}} \cos \psi I_{v}^{\prime}\left(\gamma_{2} \rho_{b}\right),
\end{array}
$$




$$
\begin{aligned}
& y_{5}=\left(\sin \psi+\frac{v \beta}{\gamma_{3}^{2} \rho_{b}} \cos \psi\right) K_{v}\left(\gamma_{3} \rho_{b}\right), \\
& y_{6}=\frac{i \omega \mu_{0}}{\gamma_{3}} \cos \psi K_{v}^{\prime}\left(\gamma_{3} \rho_{b}\right) \text {, } \\
& y_{7}=\left(\cos \psi-\frac{v \beta}{\gamma_{2}^{2} \rho_{b}} \sin \psi\right) K_{v}\left(\gamma_{2} \rho_{b}\right), \\
& y_{8}=\left(\cos \psi-\frac{v \beta}{\gamma_{2}^{2} \rho_{b}} \sin \psi\right) I_{v}\left(\gamma_{2} \rho_{b}\right) \text {, } \\
& y_{9}=\frac{i \omega \mu_{0}}{\gamma_{2}} K_{v}^{\prime}\left(\gamma_{2} \rho_{b}\right), \\
& y_{10}=\frac{i \omega \mu_{0}}{\gamma_{2}} I_{v}^{\prime}\left(\gamma_{2} \rho_{b}\right), \\
& y_{11}=\left(\cos \psi-\frac{v \beta}{\gamma_{3}^{2} \rho_{b}} \sin \psi\right) K_{v}\left(\gamma_{3} \rho_{b}\right) \text {, } \\
& y_{12}=\frac{i \omega \mu_{0}}{\gamma_{3}} \sin \psi K_{v}^{\prime}\left(\gamma_{3} \rho_{b}\right) \text {, } \\
& y_{13}=\frac{i \omega \varepsilon_{2}}{\gamma_{2}} \cos \psi K_{v}^{\prime}\left(\gamma_{2} \rho_{b}\right) \text {, } \\
& y_{14}=\frac{i \omega \varepsilon_{2}}{\gamma_{2}} \cos \psi K_{v}^{\prime}\left(\gamma_{2} \rho_{b}\right), \\
& y_{15}=\left(\sin \psi+\frac{\nu \beta}{\gamma_{2}^{2} \rho_{b}} \cos \psi\right) K_{v}\left(\gamma_{2} \rho_{b}\right) \text {, } \\
& y_{16}=\left(\sin \psi+\frac{v \beta}{\gamma_{2}^{2} \rho_{b}} \cos \psi\right) I_{\nu}\left(\gamma_{2} \rho_{b}\right) \text {, } \\
& y_{17}=\frac{i \omega \varepsilon_{3}}{\gamma_{3}} \cos \psi K_{v}^{\prime}\left(\gamma_{3} \rho_{b}\right), \\
& y_{18}=\left(\sin \psi+\frac{\nu \beta}{\gamma_{3}^{2} \rho_{b}} s \cos \psi\right) K_{v}\left(\gamma_{3} \rho_{b}\right) \text {. }
\end{aligned}
$$

Equation (11) is the eigenvalue equation corresponding to the HCLCOF structure, solutions to which will provide the modes sustained in the guide.

\section{Results and Discussion}

The two types of fiber structures, namely, the LCOF and the HCLCOF, can now be analyzed. In the case of LCOF, the relative distributions of power in the three different regions of the guide are considered, whereas the analyses corresponding to HCLCOF are limited to the modal dispersion relations and the field cutoffs. Figures 3(a), 3(b), and 3(c), respectively, illustrate the nature of power propagation in the LCOF core, inner clad, and the outer clad regions, as plotted against the propagation constant $\beta$ corresponding to the situation of TE mode excitation in the guide. In our illustrative cases, we consider four different values of core radius for the computational purpose, namely, $5 \mu \mathrm{m}, 15 \mu \mathrm{m}, 30 \mu \mathrm{m}$, and $50 \mu \mathrm{m}$, and the clad radius is kept fixed at $125 \mu \mathrm{m}$. Further, the RI values of core and the inner clad regions are taken to be $n_{1}=1.462$ and $n_{2}=1.458$, respectively, and the operating wavelength $\lambda_{0}$ is kept fixed as $633 \mathrm{~nm}$. The outermost region of the guides (LCOF and HCLCOF) is considered to be the nematic liquid crystal named as BDH mixture 14616, which has the ordinary and the extraordinary RI values as $n_{0}=$ 1.457 and $n_{e}=1.5037$, respectively.

Now, focussing on the computations of the relative distribution of power in LCOF, we observe from Figure 3(a) that the confinement of power shows an initial increase with the propagation constant $\beta$ corresponding to almost all the fiber core dimensions considered here. However, the increase is more prominent in the case of LCOFs of larger core radius. We further find that, near the higher $\beta$-tail, power confinements are observed to be increasing with that of the core dimensions, which is very much expected as the fibers of larger dimensions essentially possess the capability of confining more amount of power in the fiber core. In the lower $\beta$-tail regime, we observe some fluctuations in power, and corresponding to $50 \mu \mathrm{m}$ core radius, almost $59 \%$ of power is confined into the core section. In the medium range of the allowed propagation constants, the confinement patterns exhibit a kind of uniformity corresponding to lower dimensions of the LCOF. The largest core radius chosen here is $50 \mu \mathrm{m}$, and the confinement shows more fluctuations corresponding to this case. This is attributed to the fact that the LCOF becomes extremely multimode in this case, resulting thereby some sort of modal interference, which affects the uniformity in the distribution of power. The feature related to the kind of uniform power distribution over propagating modes is more required in telecommunication purposes, and LCOFs of smaller dimensions would possibly meet the requirements. However, more promising needs of LCOFs are expected to be in optical sensing or other integrated optic applications, which would generally require more amount of power to be confined in the outermost clad. Figure 3(a) shows the confinement to be almost about 15\%$25 \%$ distributed over major portion of the allowed values of propagation constants.

Figures 3(b) and 3(c), respectively, correspond to the relative distribution of power in the LCOF inner dielectric and the outer liquid crystal clad sections. We observe that the power confinement patterns are almost similar in both of these sections, and also, the trends of power patterns are just opposite to what we observed in the LCOF core, which is quite expected as the way confinement increases or decreases (with the propagation constant) in the core section, it will decrease or increase, respectively, in the two clads. The noticeable fact remains that the average value of power in the two clad sections is appreciably higher (within the longer $\beta$-range) than what is observed in the LCOF core. More precisely, corresponding to lower LCOF core dimensions, within the uniform range of the allowed values of propagation constants, approximately $45 \%$ power is shared by each of the clad regions, whereas around only $10 \%$ power transmission is observed in the LCOF core at the same time. This essentially indicates a substantial increase of power in 


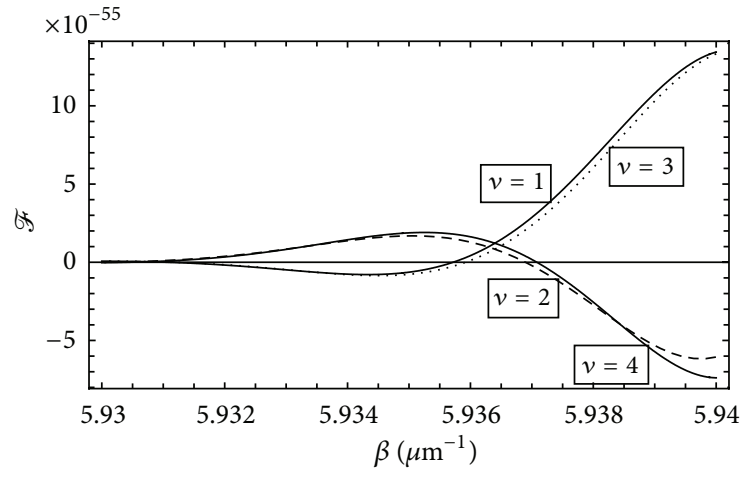

(a)

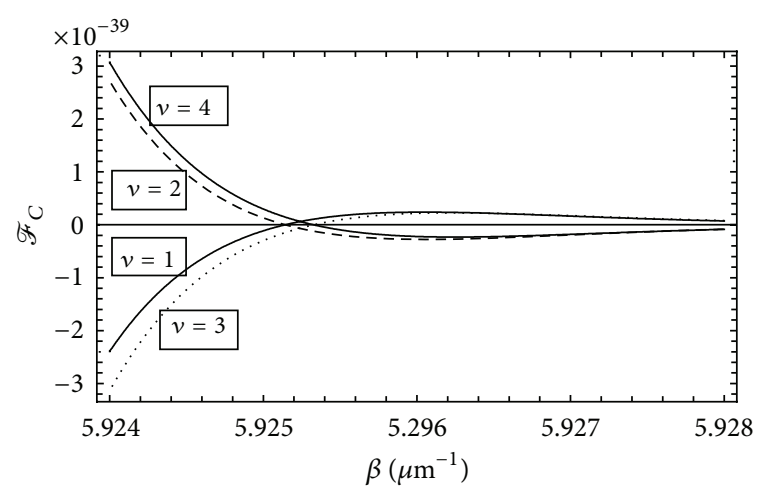

(b)

FIGURE 4: (a) Plot of the dispersion relation for the TE modes corresponding to $\psi=0^{\circ}$. (b) Plot of the cutoff characteristics for the TE modes corresponding to $\psi=0^{\circ}$.

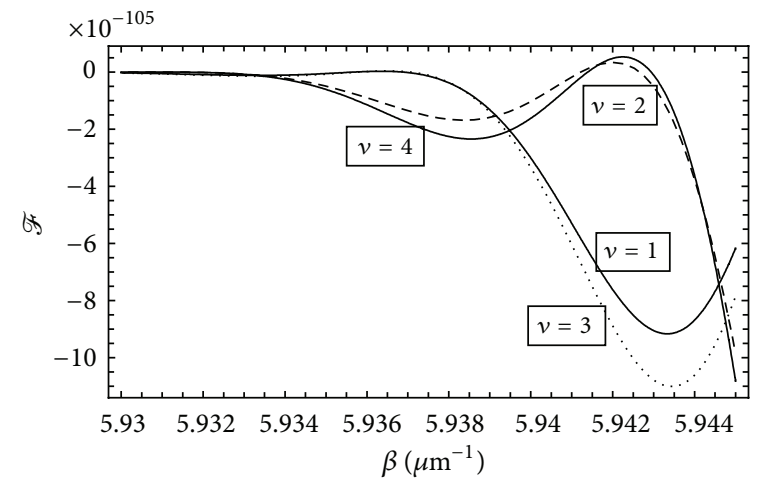

(a)

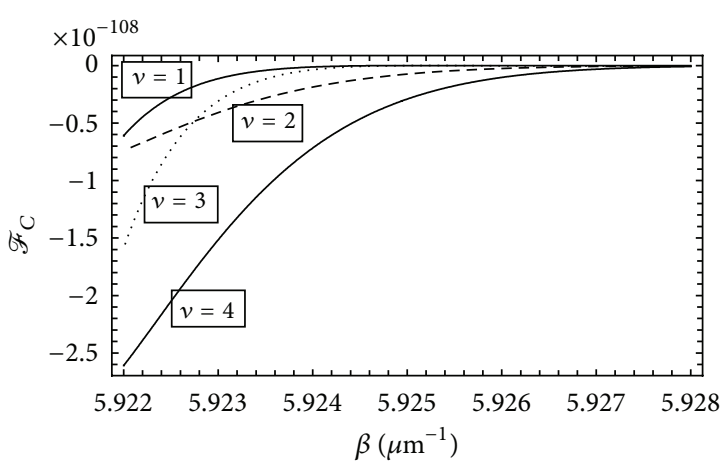

(b)

FIgURE 5: (a) Plot of the dispersion relation for the TE modes corresponding to $\psi=90^{\circ}$. (b) Plot of the cutoff characteristics for the TE modes corresponding to $\psi=90^{\circ}$.

the two clad sections, which is attributed to the presence of nematic radially anisotropic liquid crystal material in the outermost clad region. The distribution of power, however, does not seem to be that uniform in the lower $\beta$-tail regime, and the uniformity is more affected with the increase in core dimension owing to the reason of possibilities related to modal interference in this case. Finally, the combined observations of the uniformity of power patterns corresponding to the LCOFs of smaller dimensions and higher power confinements in the outermost liquid crystal section (Figures $3(\mathrm{a})$ and 3(b)) essentially indicate the possibility of useful integrated optic applications of these guides, for example, in field coupling and/or optical sensing needs.

Now, turning the attention towards the case of HCLCOFs, we make an attempt to study the dispersion relation expressed by (11). In this stream, we consider the values of the fiber core radius as $60.38 \mu \mathrm{m}$ and the inner clad radius as $120.07 \mu \mathrm{m}$. The operating wavelength is chosen as $1.55 \mu \mathrm{m}$, and the RI values of the core and the inner clad sections are taken to be 1.5 and 1.46, respectively. The physical properties of the outermost liquid crystal section are considered to be the same as in the case of LCOF discussed above. Equation (11) is plotted corresponding to four different values of the azimuthal index $v$, namely, $v=1, v=2, v=3$, and $v=4$, and the introduced conducting helical turns may have perpendicular or parallel orientations with respect to the optical axis, making thereby the respective helical pitch angles as $\psi=0^{\circ}$ and $\psi=90^{\circ}$.

Figure 4(a) illustrates the plots of the left hand side $\mathscr{F}$ of (6) in the case when the helix pitch angles are perpendicular to the optical axis, that is, $\psi=0^{\circ}$. In this case, we observe that, corresponding to all values of the azimuthal indices, the plots intersect the $\mathscr{F}=0$ axis; the points of intersection essentially determine the propagation constant of the corresponding mode. We observe that the modal propagation constants corresponding to $v=1$ and $v=3$ are very close, though with the situation $\left.\beta)_{v=1} \leq \beta\right)_{v=2}$. Further, the modes with $v=2$ and $v=4$ attain almost similar $\beta$-values with $\left.\beta)_{v=2} \leq \beta\right)_{v=4}$.

Figure 4(b) describes the situation when (11) is solved under the limit $\gamma_{2}^{2} \rightarrow 0$, indicating thereby the respective plots (of $\mathscr{F}_{C}$ ) to correspond the case of field cutoffs. We observe that the cutoffs for the modes with $v=1$ and $v=3$ are almost the same, and that with $v=2$ and $v=4$ too are very much similar. However, we observe from Figures 4(a) and 4(b) that the cutoff $\beta$-values, as seen in Figure 4(b), are less than that obtained from the knowledge of 
$\beta$-values noticed from the intersections in Figure 4(a), which essentially concludes that the results illustrated in Figures 4(a) and 4(b) are consistent.

It is noteworthy that the helix pitch angle $\psi$ introduced in HCLCOF has the ability to alter the dispersion characteristics of the guide. In order to demonstrate this, plots are made corresponding to $90^{\circ}$ pitch, and those corresponding to the dispersion equation and the field cutoffs are illustrated in Figures 5(a) and 5(b). In both these figures, profound effect due to helix pitch can clearly be observed. It is found that the $\beta$-values are roughly increased in this case. Making the pitch $\psi=90^{\circ}$ leaves the impression of eliminating the helical turns as those are now oriented just parallel to the optical axis. Thus, it can be conceptually understood that the presence of conducting helical turns (i.e., $\psi=0^{\circ}$ ) introduces the modal propagation constants to be lower. It has been reported before that helix pitch makes the propagating waves to attenuate up to some extent-the property which can clearly be seen in the case of HCLCOF as the waves propagate with lower propagation constants under the perpendicular orientation (with respect to the optical axis) of helical turns.

\section{Conclusion}

From the foregoing discussions, inference can be drawn that, under TE mode excitation, LCOFs present higher amount of power confinement in the inner dielectric and the outer liquid crystal clads-the feature that can be effectively used in several integrated optic applications including electromagnetic field coupling and optical sensing. It has been found that, with the increase in propagation constants, the confinement patterns become almost uniform, which is not observed corresponding to LCOFs of higher core diameters. Higher confinement of power in the cladding regions can be interpreted as if the power is leaking off the LCOF core, and this feature can be attributed to the presence of radially anisotropic liquid crystal material in the outermost section of the guide.

Furthermore, the fundamental investigations of HCLCOFs reveal that the introduction of conducting helical windings at the interface of the core-inner dielectric clad sections makes the guide to attain the attenuation property. The possibility of suitable adjustments of the helix pitch leaves the option to alter the attenuation characteristics of the guide, the feature quite indispensable in several optical applications.

\section{Acknowledgment}

The author gratefully acknowledges the financial support given by the Ministry of Higher Education (Malaysia) through the Fundamental Research Grant project no. (FRGS/ 1/2011/TK/UKM/01/16).

\section{References}

[1] A. Jákli, D. Krüerke, and G. G. Nair, "Liquid crystal fibers of bent-core molecules," Physical Review E, vol. 67, no. 5, Article ID 051702, 6 pages, 2003.
[2] T. R. Wolinski, K. Szaniawska, S. Ertman et al., "Influence of temperature and electrical fields on propagation properties of photonic liquid-crystal fibres," Measurement Science and Technology, vol. 17, no. 5, pp. 985-991, 2006.

[3] T. R. Wolinski, S. Ertman, A. Czapla et al., "Polarization effects in photonic liquid crystal fibers," Measurement Science and Technology, vol. 18, pp. 3061-3069, 2007.

[4] B. P. Cumming, A. Jesacher, M. J. Booth, T. Wilson, and M. $\mathrm{Gu}$, "Adaptive aberration compensation for three-dimensional micro-fabrication of photonic crystals in lithium niobate," Optics Express, vol. 19, pp. 9419-9425, 2011.

[5] Y. Fu, F. Barnes, T. Ewing, S. Serati, and X. W. Xia, "Liquid crystal modulated optical CDMA in a fiber-based testbed," in Active and Passive Optical Components for WDM Communications IV, vol. 5595 of Proceedings of SPIE, pp. 302-309, October 2004.

[6] S. T. Wu and U. Efron, "Optical properties of thin nematic liquid crystal cells," Applied Physics Letters, vol. 48, pp. 624-636, 1986.

[7] I. Sage and D. Chaplin, "Low RI liquid crystals for integrated optics," Electronics Letters, vol. 23, no. 22, pp. 1192-1193, 1987.

[8] M. Green and S. J. Madden, "Low loss nematic liquid crystal cored fiber waveguides,” Applied Optics, vol. 28, pp. 5202-5203, 1989.

[9] H. Lin, P. P. Muhoray, and M. A. Lee, "Liquid crystalline cores foroptical fibers," Molecular Crystals and Liquid Crystals, vol. 204, pp. 189-200, 1991.

[10] C. Veilleux, J. Lapierre, and J. Bures, "Liquid-crystal-clad tapered fibers," Optics Letters, vol. 11, pp. 733-735, 1986.

[11] S. H. Chen and T. J. Chen, "Observation of mode selection in a radially anisotropic cylindrical waveguide with liquid-crystal cladding," Applied Physics Letters, vol. 64, no. 15, pp. 1893-1895, 1994.

[12] Z. K. Ioannidis, I. P. Giles, and C. Bowry, "All-fiber optic intensity modulators using liquid crystals," Applied Optics, vol. 30, pp. 328-333, 1991.

[13] E. S. Goldburt and P. S. J. Russell, "Electro-optical response of a liquid-crystalline fiber coupler," Applied Physics Letters, vol. 48, no. 1, pp. 10-12, 1986.

[14] T. Yoshino, Y. Takahashi, H. Tamura, and N. Ohde, "Some special fibers for distributed sensing of uv light, electric field or strain," in Distributed and Multiplexed Fiber Optic Sensors III, vol. 2071 of Proceedings of SPIE, pp. 242-254, December 1993.

[15] P. K. Choudhury and W. K. Soon, "On the transmission by liquid crystal tapered optical fibers," Optik, vol. 122, no. 12, pp. 10611068, 2011.

[16] J. R. Pierce, Travelling Wave Tubes, D. Van Nostrand, Princeton, NJ, USA, 1950.

[17] C. C. Siong and P. K. Choudhury, "Propagation characteristics of tapered core helical cald dielectric optical fibers," Journal of Electromagnetic Waves and Applications, vol. 23, no. 5, pp. 663674, 2009.

[18] A. H. B. M. Safie and P. K. Choudhury, "On the hybrid field patterns of helical clad dielectric optical fibers," Progress in Electromagnetics Research, vol. 91, pp. 69-84, 2009.

[19] K. Y. Lim, P. K. Choudhury, and Z. Yusoff, "Chirofibers with helical windings - an analytical investigation," Optik, vol. 121, no. 11, pp. 980-987, 2010.

[20] P. K. Choudhury and D. Kumar, "On the slow-wave helical clad elliptical fibers," Journal of Electromagnetic Waves and Applications, vol. 24, no. 14-15, pp. 1931-1942, 2010.

[21] A. W. Snyder and F. Rühl, "Single-mode, single-polarization fibers made of birefringent material," Journal of the Optical Society of America, vol. 73, pp. 1165-1174, 1983. 
[22] Y. Chen, "Anisotropic fiber with cylindrical polar axes," Applied Physics B, vol. 42, no. 1, pp. 1-3, 1987.

[23] A. H. Cherin, An Introduction to Optical Fibers, chapter 5, McGraw-Hill, New York, NY, USA, 1987.

[24] D. A. Watkins, Topics in Electromagnetic Theory, Wiley, New York, NY, USA, 1958. 

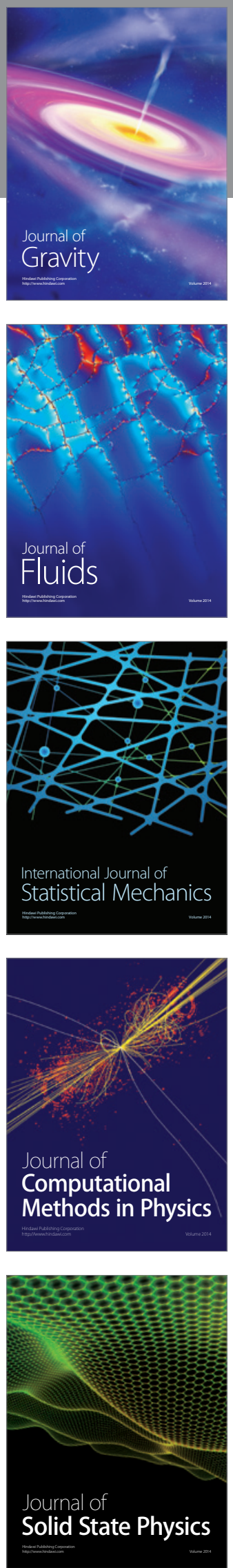

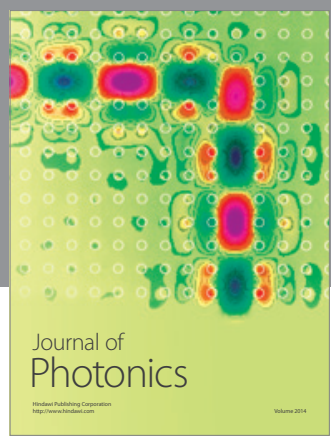

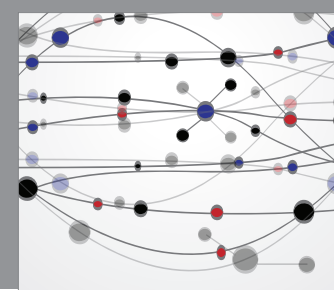

The Scientific World Journal

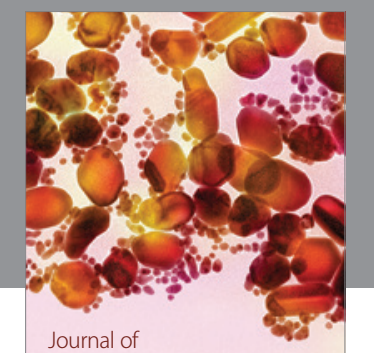

Soft Matter
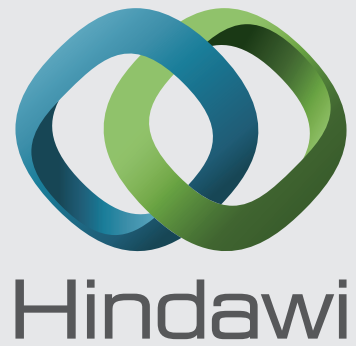

Submit your manuscripts at

http://www.hindawi.com
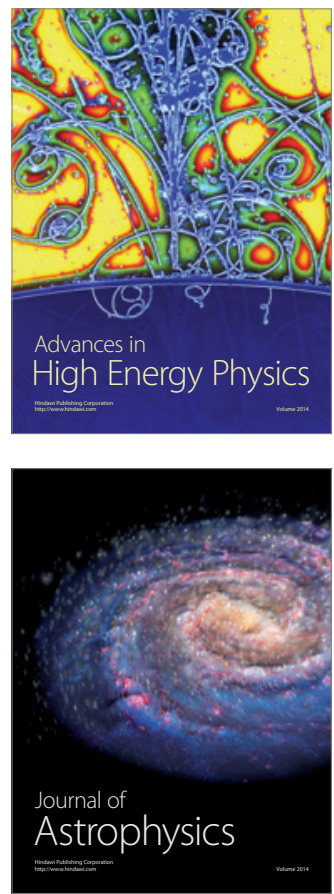
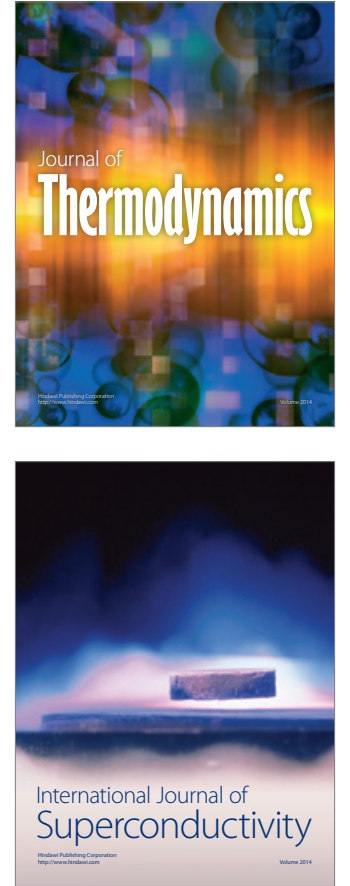
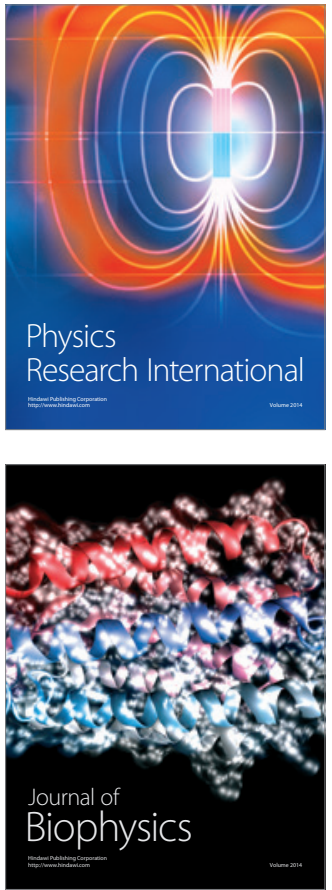
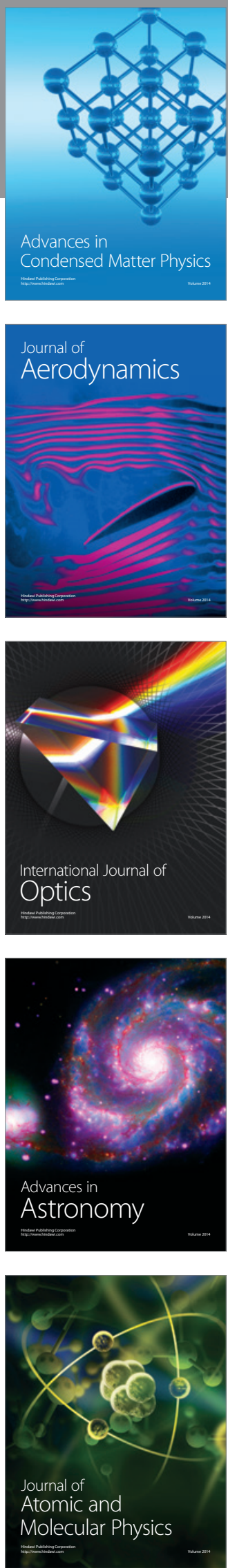